\title{
Identification of chemical constituents of Selaginella doederleinii Hieron
}

\author{
Korbanjhon Brad ${ }^{1, a}$,Yan Zhang ${ }^{2 *, b}$, Xu Yang ${ }^{2, c}$,Tianxin Wang ${ }^{2, d}$ \\ ${ }^{1}$ College of Chemistry and Environmental Sciences, Yili Normal University, Yining, 835000, China, \\ ${ }^{2}$ Key Laboratory of Food Nutrition and Safety (Tianjin University of Science and Technology), \\ Ministry of Education, Tianjin 300457, China \\ akorbanjhon@126.com, ${ }^{\mathrm{b}} \mathrm{cpzy}$ yan@126.com, \\ cxy1009@tust.edu.cn, ${ }^{\mathrm{c}}$ wtxwang@tust.edu.cn
}

\begin{abstract}
Keywords: Selaginella doederleinii Hieron, chemical constituents, structure identification, spectroscopy
\end{abstract}

Abstract. To determine the chemical constituents of Selaginella doederleinii Hieron.

The compounds were isolated and purified from the Ethyl Acetate Fraction from Ethanol Extract of Selaginella doederleinii Hieron by multiple chromatographic techniques including macroporous resin, polyamide, silica gel, Sephadex LH-20 and pre-HPLC. The structures were identified on the basis of thierphysico-chemical properties and spectroscopic data analyses. Twenty compounds were isolated and determined as myristic acid (compound 1), $\beta$-Citronellol (Compound 2), palmitic acid (Compound 3), stearic acid (Compound 4), beta-sitosterol(Compound 5), physcion(Compound 6), 3-beta -Acetoxysitost-5-en-7-one(Compound 7), emodin(Compound 8), chrysophanol(Compound 9), apigenin(Compound 10), amentoflavone(Compound 11), robustaflavone 7,4',7"-o-trimethyl ether(Compound 12), heveaflavone(Compound 13), podocarpusflavone A(Compound 14), robustaflavone 4',4"'-o-dimethyl ether(Compound 15), robustaflavone 4'-o-methyl ether(Compound 16), adenosine(Compound 17), ferulic acid(Compound 18), syringate(Compound 19) and vanillic acid(Compound 20) according to physical and chemical properties and spectral data. Among those compounds, compound 2, compound 6, compound 9, compound 17, compound 19, compound 20 were firstly isolated from this plant.

\section{Introduction}

The genus Selaginella has 700 species, about 50 species of them exist in China .Six species are used as traditional Chinese medicine, and one of them is Selaginella doederleinii Hieron .Selaginella doederleinii Hieron is widely distributed in the South of China and recorded in Chinese Pharmacopoeia. Many Evidences demonstrated that Selaginella doederleinii Hieron has many pharmacologic effects including blood glucose and blood lipid modulation, antioxidation, antivirus, antitumor activities [1-3]. Previous chemical investigations of this plant have confirmed the presence of biflavonoids, lignans, and alkaloids. However, at present, little work has been done on the chemical composition of Selaginella doederleinii Hieron. Therefore, in this research, we isolated and identified the chemical components of Selaginella doederleinii Hieron extracts.

\section{Experimental}

\section{Materials and equipment}

NMR spectra were measured on a Varian INOVA-500 NMR spectrometer. Column chromatography was carried out on silica gel (100 to 200 mesh and 200 to 300 mesh) and GF254 Silica gel that were products of Qingdao Marine Chemistry Co. Ltd. Sephadex LH-20 was the 
product of Sweden Pharmacia Biotech. All the chemicals and solvents used in the experiment were of analytically pure agent.

\section{Plant materials}

Selaginella doederleinii Hieron was collected in GuiLin, GuangXi, China in the september of 2014. The material was confirmed by Prof. Korbanjhon Brad in Yili Normal University (The School of Chemistry \& Environmental Engineering) and was preserved with the identification number of YLNU2014090902 in the specimen preservation site of the university.

\section{Extraction and isolation of compounds from Selaginella doederleinii Hieron}

$25 \mathrm{~kg}$ of Selaginella doederleinii Hieron was crushed into powder and extracted with $200 \mathrm{~L}$ of $95 \%$ ethanol for 3 times. The ethanol extract solution was filtered and concentrated under reduced pressure to give $700 \mathrm{~g}$ of the extract. The extract was suspended in water and then sequentially extracted with petroleum ether, ethyl acetate and butanol. The extracts were evaporated under reduced pressure to obtain $220 \mathrm{~g}$ of ethyl acetate extract.

The ethyl acetate extract $(220 \mathrm{~g})$ was subjected to silica gel column chromatography and eluted gradiently with petroleum ether-ethyl acetate $(6: 1,4: 1,2: 1,1: 1,1: 2$ and 1:3), EtOAc, EtOAc $(5 \% \mathrm{MeOH})$, EtOAc $(10 \% \mathrm{MeOH})$, EtOAc $(20 \% \mathrm{MeOH})$ and $\mathrm{MeOH}$. Subsequently, 7 fractions(Fr1, Fr2, Fr3, Fr4, Fr5, Fr6and Fr7) were obtained. By purifying with silica gel column chromatography and Sephadex LH-20 column chromatography, 20 compounds were obtained. Among them, compound 1,compound 2, compound 3 and compound 4 were isolated from Fr1; compound 5 and compound 6 were isolated from Fr2; compound 7 were isolated from Fr3; compound 8, compound 9 and compound 10 were isolated from Fr4; compound 11 and compound 12 were isolated from Fr5; compound 13,compound 14, compound 15 and compound 16 were isolated from Fr6; compound 17,compound 18, compound 19 and compound 20 were isolated from Fr7.

\section{Results}

7 fractions (Fr1, Fr2, Fr3, Fr4, Fr5, Fr6and Fr7) were obtained. And 20 compounds were separated and identified according to physical and chemical properties and spectral data.

Based on the previous reports and spectroscopic data, compound 1(8mg),compound 2(6mg), compound 3(13mg) and compound 4(7mg)in Fr1 were identified as Myristic acid, $\beta$-Citronellol , palmitic acid and stearic acid, respectively. compound 5(27mg) and compound 6(12mg) in Fr2 were identified as $\beta$-sitosterol and Physcion; compound $7(10 \mathrm{mg})$ in Fr3 was identified as $3 \beta$-Acetoxysitost-5-en-7-one; compound $8(7 \mathrm{mg})$, compound $9(11 \mathrm{mg})$ and compound $10(15 \mathrm{mg})$ in Fr4 were identified as emodin, Chrysophanol, Apigenin. compound 17(8mg),compound 18(6.7mg), compound 19(9mg) and compound 20(5mg)in Fr7 were identified as Adenosine, ferulic acid, Syringate and Vanillic acid, respectively.The compounds from Fr 5 and 6 were identified as flavonoids as following:

Compound 11(13 mg), mp $>300^{\circ} \mathrm{C},{ }^{1} \mathrm{H}-\mathrm{NMR}\left(\mathrm{C}_{5} \mathrm{D}_{5} \mathrm{~N}\right) \delta: 7.88\left(1 \mathrm{H}, \mathrm{d}, J=2 \mathrm{~Hz}, \mathrm{H}-2^{\prime}\right), 7.73(1 \mathrm{H}, \mathrm{dd}$, $J=2 \mathrm{~Hz}, \mathrm{H}-6$ '), 7.41 (2H, d, J=9Hz, H-2'", H-6"'), 7.01 (1H, d, J=9Hz, H-5'), 6.65 (2H, d, J=9Hz, H-3"', H-5"'), 6.48 (2H, s, H-3, H-3'), 6.09 (1H, d, J=2Hz, H-6"), 6.35 (1H, d, J=2Hz, H-8), 6.29 $(1 \mathrm{H}, \mathrm{s}, \mathrm{H}-6) ;{ }^{13} \mathrm{C}-\mathrm{NMR}\left(\mathrm{C}_{5} \mathrm{D}_{5} \mathrm{~N}\right) \delta: 184.0(\mathrm{C}-4 "), 183.6$ (C-4), 166.7 (C-2), 165.6 (C-2"), 165.5 (C-7), 164.7 (C-7"), 164.0 (C-4"'), 163.6 (C-5"), 163.3 (C-5), 162.2 (C-4'), 159.4 (C-9), 156.8 (C-9"), 133.5 (C-6'), 129.8 (C-2"', C-6"'), 129.3 (C-2'), 123.4 (C-3'), 123.3 (C-1'), 122.7 (C-1"'), 118.2 (C-5'), 117.8 (C-3"', 5"'), 106.2(C-10), 106.0 (C-10"), 105.9(C-8"), 105.0 (C-3), 104.5 (C-3"), 100.9 (C-6"), 100.7 (C-6), 95.8 (C-8). The physicochemical properties and spectroscopic data were consistent with the reference [4]. Compound 11 was identified as amentoflavone. 
Compound $12(7.4 \mathrm{mg}), \mathrm{mp}>300^{\circ} \mathrm{C},{ }^{1} \mathrm{H}-\mathrm{NMR}\left(\mathrm{C}_{5} \mathrm{D}_{5} \mathrm{~N}\right) \delta: 13.09(1 \mathrm{H}, \mathrm{s},-\mathrm{OH}), 12.78(1 \mathrm{H}, \mathrm{s},-\mathrm{OH})$, $10.60(1 \mathrm{H}, \mathrm{s},-\mathrm{OH}), 8.21$ (2H, d, J=9.0 Hz, H-2'"'/6"'), 8.12 (1H, dd, J=2.0, 9.0 Hz, H-6'), 7.97 (1H, d, $J=2 \mathrm{~Hz}, \mathrm{H}-2$ '), 7.26 (1H, d, J=9Hz, H-5'), 7.22 (2H, d, J=9Hz, H-3'"/5'"), 7.12 (1H, s, H-3), 6.98 (1H, s, H-3"), $6.97(1 \mathrm{H}, \mathrm{d}, J=2 \mathrm{~Hz}, \mathrm{H}-8), 6.51(1 \mathrm{H}, \mathrm{d}, J=2 \mathrm{~Hz}, \mathrm{H}-5), 6.42\left(1 \mathrm{H}, \mathrm{s}, \mathrm{H}-8^{\prime \prime}\right), 3.90(3 \mathrm{H}, \mathrm{s}$, OMe-4'), 3.88 (3H, s, OMe-4"'), 3.81 (3H, s, OMe-7); ${ }^{13} \mathrm{C}-\mathrm{NMR}\left(\mathrm{C}_{5} \mathrm{D}_{5} \mathrm{~N}\right) \delta: 184.0(\mathrm{C}-4 "), 183.7$ (C-4), 164.8 (C-2), 165.1 (C-2"), 166.9 (C-7), 163.8 (C-7"), 105.1 (C-3), 105.7 (C-3"), 162.4 (C-5"), 163.4(C-5), 112.8 (C-6"), 101.0 (C-6), 95.9 (C-8"), 95.9 (C-8), 159.5 (C-9), 156.5 (C-9"), 106.0 (C-10), 105.8 (C-10"), 124.4 (C-1'), 124.1 (C-1'"), 133.0 (C-2'), 129.4 (C-2"'), 125.0 (C-3'), 115.9 (C-3"'), 164.1 (C-4'), 164.4 (C-4'"), 112.8 (C-5'), 115.9 (C-5"'), 129.4 (C-6'), 129.2 (C-6"'), 57.0 (OMe-4"), 56.4 (OMe-4'"), 57.0 (OMe-7). The physicochemical properties and spectroscopic data were consistent with the reference[6]. Compound 12 was identified as robustaflavone 7,4',7"-o-trimethyl ether.

Compound $13(15 \mathrm{mg}), \mathrm{mp}>300^{\circ} \mathrm{C},{ }^{1} \mathrm{H}-\mathrm{NMR}\left(\mathrm{C}_{5} \mathrm{D}_{5} \mathrm{~N}\right) \delta: 8.04\left(1 \mathrm{H}, \mathrm{dd}, J=8.6,2.2 \mathrm{~Hz}, \mathrm{H}-66^{\prime}\right), 8.02(1 \mathrm{H}$, $\mathrm{d}, J=2.2 \mathrm{~Hz}, \mathrm{H}-2 '), 7.66\left(2 \mathrm{H}, \mathrm{d}, J=8.9 \mathrm{~Hz}, \mathrm{H}-2^{\prime \prime}, 6^{\prime \prime}\right), 7.16(1 \mathrm{H}, \mathrm{d}, J=8.6 \mathrm{~Hz}, \mathrm{H}-5$ '), 6.94(1H, s, H-3), $6.92\left(2 \mathrm{H}, \mathrm{d}, J=8.9 \mathrm{~Hz}, \mathrm{H}-3^{\prime \prime \prime}, 5^{\prime \prime}\right), 6.91(1 \mathrm{H}, \mathrm{s}, \mathrm{H}-3 "), 6.77(1 \mathrm{H}, \mathrm{d}, J=2.2 \mathrm{~Hz}, \mathrm{H}-8), 6.68$ (1H, s, H-6"), 6.36 (1H, d, J=2.2Hz, H-6), 3.84 (3H, s, OMe-7), 3.82 (3H, s, OMe-7"), 3.75 (3H, s, OMe-4"'); ${ }^{13} \mathrm{C}-\mathrm{NMR}\left(\mathrm{C}_{5} \mathrm{D}_{5} \mathrm{~N}\right) \quad \delta: 184.0(\mathrm{C}-4 "), 183.7(\mathrm{C}-4), 165.6(\mathrm{C}-2), 166.8(\mathrm{C}-2 "), 164.7(\mathrm{C}-7), 163.6$ (C-7"), 105.1 (C-3), 105.9(C-3"), 162.3 (C-5"), 163.4 (C-5), 110.9 (C-6"), 100.9 (C-6), 95.8 (C-8"), 95.8 (C-8), 159.5 (C-9), 156.8 (C-9"), 106.2 (C-10), 106.0 (C-10"), 125.0 (C-1'), 123.4 (C-1"'), 133.5 (C-2'), 129.8 (C-2'"), 125.0 (C-3'), 117.8 (C-3"'), 164.0 (C-4'), 163.2 (C-4'"), 112.8 (C-5'), 118.2 (C-5"'), 129.3 (C-6'), 129.9 (C-6'"), 56.9 (OMe). The physicochemical properties and spectroscopic data were consistent with the reference [4]. Compound 13 was identified as heveaflavone.

Compound $14(6 \mathrm{mg}), \mathrm{mp}>300^{\circ} \mathrm{C},{ }^{1} \mathrm{H}-\mathrm{NMR}\left(\mathrm{C}_{5} \mathrm{D}_{5} \mathrm{~N}\right) \delta: 13.14(1 \mathrm{H}, \mathrm{s}, \mathrm{OH}-5), 13.01$ (1H, s, OH-5"), $8.13\left(1 \mathrm{H}, \mathrm{d}, J=2.4 \mathrm{~Hz}, \mathrm{H}-2^{\prime}\right), 8.03\left(1 \mathrm{H}, \mathrm{dd}, J=2.4 \mathrm{~Hz}, 8.6 \mathrm{~Hz}, \mathrm{H}-6{ }^{\prime}\right), 7.72$ (2H, d, J=9.1 Hz, H-2'"), 7.24(1H, d, $\left.J=8.6 \mathrm{~Hz}, \mathrm{H}-5^{\prime}\right), 6.92$ (2H, d, $\left.J=9.1 \mathrm{~Hz}, \mathrm{H}-3^{\prime \prime \prime}\right), 6.72$ (1H, s, H-3), 6.70 (1H, s, H-3"), 6.50 (1H, d, $J=2.1 \mathrm{~Hz}, \mathrm{H}-8), 6.44$ (1H, s, H-6"), 6.23 (1H, d, J=2.1 Hz, H-6), 3.78 (3H, s, OMe-4"'). ${ }^{13} \mathrm{C}-\mathrm{NMR}\left(\mathrm{C}_{5} \mathrm{D}_{5} \mathrm{~N}\right) \delta$ : 183.8 (C-4"), 183.7 (C-4), 164.6 (C-2), 165.4(C-2"), 166.8 (C-7), 163.6 (C-7"), 105.1 (C-3), 105.7 (C-3"), 163.6 (C-5"), 164.0 (C-5), 112.8 (C-6"), 101.0 (C-6), 95.9 (C-8"), 95.3 (C-8), 159.5 (C-9), 158.6 (C-9"), 106.0 (C-10), 105.8 (C-10"), 123.4 (C-1'), 124.1 (C-1'"), 132.7 (C-2'), 129.8 (C-2"'), 105.6 (C-3'), 105.9 (C-3'"), 164.1 (C-4'), 164.4 (C-4'"'), 110.9 (C-5'), 112.9 (C-5"'), 129.9 (C-6'), 129.9 (C-6"'), 56.9 (OMe-4"'). The physicochemical properties and spectroscopic data were consistent with the reference [5]. Compound 14 was identified as podocarpusflavone A.

Compound $15(8 \mathrm{mg}), \mathrm{mp}>300^{\circ} \mathrm{C},{ }^{1} \mathrm{H}-\mathrm{NMR}\left(\mathrm{C}_{5} \mathrm{D}_{5} \mathrm{~N}\right) \delta: 13.11(1 \mathrm{H}, \mathrm{s}, \mathrm{OH}-5 "), 12.81(1 \mathrm{H}, \mathrm{s}, \mathrm{OH}-5)$, 10.70 (1H, s, OH-7), 10.53 (1H, s, OH-7"), 8.19 (1H, dd, J=2.0, 9.0 Hz, H-6'), 8.14 (2H, d, J=9.0 Hz, H-2"'/6'"), 7.89 (1H, d, J=2.0 Hz, H-2'), 7.40 (1H, d, J=9.0 Hz, H-5'), 7.23 (2H, d, J=9.0 Hz, H-3"'/5"'), 6.94 (1H, s, H-3"), 6.93 (1H, s, H-3), 6.66 (1H, d, J=2 Hz, H-8), 6.37 (1H, s, H-8"), 6.33 $(1 \mathrm{H}, \mathrm{d}, J=2 \mathrm{~Hz}, \mathrm{H}-5), 3.91$ (6H, s, OMe-4'/4'"). ${ }^{13} \mathrm{C}-\mathrm{NMR}\left(\mathrm{C}_{5} \mathrm{D}_{5} \mathrm{~N}\right) \delta: 184.2(\mathrm{C}-4 "), 184.0$ (C-4), 164.4 (C-2), 164.9 (C-2"), 167.2 (C-7), 165.8 (C-7"), 106.2 (C-3), 106.3 (C-3"), 159.7 (C-5"), 162.4 (C-5), 111.3 (C-6"), 101.3 (C-6), 95.7 (C-8"), 96.3 (C-8), 159.8 (C-9), 159.0 (C-9"), 105.9 (C-10), 105.7 (C-10"), 123.3 (C-1'), 123.6 (C-1"'), 133.1 (C-2'), 130.2 (C-2"'), 123.6 (C-3'), 114.7 (C-3'"), 164.0 (C-4'), 165.6 (C-4'"), 113.2 (C-5'), 118.1 (C-5"'), 129.4 (C-6'), 130.2 (C-6"'), 57.3 (OMe-4'), 57.3 (OMe-4"). The physicochemical properties and spectroscopic data were consistent with the reference [6]. Compound 15 was identified as robustaflavone 4',4"'-o-dimethyl ether. 
Compound $16(10 \mathrm{mg}), \mathrm{mp}>300^{\circ} \mathrm{C},{ }^{1} \mathrm{H}-\mathrm{NMR}\left(\mathrm{C}_{5} \mathrm{D}_{5} \mathrm{~N}\right) \delta: 14.20(1 \mathrm{H}, \mathrm{s},-\mathrm{OH}), 13.91(1 \mathrm{H}, \mathrm{s},-\mathrm{OH})$, $8.18\left(1 \mathrm{H}, \mathrm{dd}, J=2.3,8.0 \mathrm{~Hz}, \mathrm{H}-6^{\prime}\right), 8.01(1 \mathrm{H}, \mathrm{d}, J=8.0 \mathrm{~Hz}, \mathrm{H}-6), 8.47$ (1H, d, J=2.3Hz, H-2'), 7.99 (1H, d, $\left.J=6.8 \mathrm{~Hz}, \mathrm{H}-2^{\prime}\right), 6.84(1 \mathrm{H}, \mathrm{d}, J=2.0 \mathrm{~Hz}, \mathrm{H}-8), 7.31\left(1 \mathrm{H}, J=8.0 \mathrm{~Hz}, \mathrm{H}-5{ }^{\prime}\right), 6.90(1 \mathrm{H}, \mathrm{d}, J=2.0 \mathrm{~Hz}$, $\mathrm{H}-6) ;{ }^{13} \mathrm{C}-\mathrm{NMR}\left(\mathrm{C}_{5} \mathrm{D}_{5} \mathrm{~N}\right) \delta: 183.1$ (C-4"), 182.9 (C-4), 164.7 (C-2), 166.1 (C-2"), 164.8 (C-7), 164.5 (C-7"), 104.2 (C-3), 104.8(C-3"), 161.7 (C-5"), 162.7 (C-5), 100.3 (C-6"), 112.2 (C-6), 94.7 (C-8"), 95.2 (C-8), 158.8 (C-9), 157.9 (C-9"), 105.3 (C-10), 105.2 (C-10"), 124.2 (C-1'), 122.3 (C-1"'), 131.9 (C-2'), 129.0 (C-2'"), 122.6 (C-3'), 117.1 (C-3"'), 163.7 (C-4'), 160.7 (C-4'"), 112.0 (C-5'), 116.8 (C-5'"), 128.4 (C-6'), 129.2 (C-6"'), 56.3 (OMe-4'). The physicochemical properties and spectroscopic data were consistent with the reference [7]. Compound 16 was identified as robustaflavone 4'-o-methyl ether.

\section{Discussion}

In the present study, the ethanol Extract of Selaginella doederleinii Hieron was suspended in water and then sequentially extracted with petroleum ether, ethyl acetate and butanol. The extracts were evaporated under reduced pressure to obtain the petroleum ether extract,the ethyl acetate extract and the butanol extract. Then these extracts were suffered with MTT method with human cancer cells to assess their anti-tumor activities in vitro. As a result, the ethyl acetate extract shows good anti-tumor activity in vitro.

So,20 compounds were isolated in Ethyl Acetate Fraction from Ethanol Extract of Selaginella doederleinii Hieron. including flavonoid, organic acid, fatty acid, sterol and so on.To our knowledge, compound 2, compound 6, compound 9, compound 17, compound 19, compound 20 were firstly isolated from this plant.

\section{Conclusions}

Selaginella doederleinii Hieron belongs to the Selaginellaceae Genus plants of Pteridophyta. It is widely used to treat sore throats, rheumatic arthralgia pain, nasopharyngeal carcinoma, chorionic carcinoma, lung cancer, cervical cancer and a variety of diseases such as traumatic bleeding[8-13]. However, its anti-tumor effect of the material basis has reported relatively less. Therefore, it is necessary to conduct in-depth study in the aspect. The ethyl acetate extract shows good anti-tumor activity in vitro with MTT method with human cancer cells.

The separation and purification of ethyl acetate extract was carried out by by multiple chromatographic techniques. Twenty compounds were successfully obtained from this extact. Their structures were identified as myristic acid (Compound 1), $\beta$-Citronellol (Compound 2), palmitic acid (Compound 3), stearic acid (Compound 4) , $\beta$-sitosterol(Compound 5), physcion(Compound 6), 3ß-Acetoxysitost-5-en-7-one(Compound 7), emodin(Compound 8), chrysophanol(Compound 9), apigenin(Compound 10), amentoflavone(Compound 11), robustaflavone 7,4',7"-o-trimethyl ether(Compound 12), heveaflavone(Compound 13), podocarpusflavone A(Compound 14), robustaflavone 4',4'"-o-dimethyl ether(Compound 15), robustaflavone 4'-o-methyl ether(Compound 16), adenosine(Compound 17), ferulic acid(Compound 18), syringate(Compound 19) and vanillic acid(Compound 20) according to physical and chemical properties and spectral data. Among them, compound 2, compound 6, compound 9, compound 17, compound 19 , compound 20 were firstly isolated from this plant. 


\section{Acknowledgements}

The Project Supported by the National Science Foundation of China (31401663), the Foundation of Tianjin University of Science and Technology, Institute for New Rural Development, P. R. China (No. XNC201511), and Youth Innovation Foundation of Tianjin University of Science and Technology (No. 20151G24).

\section{References}

[1] S.A.Li, R.H.Zhu, M.Zhong, Y.P.Zhang, K.L.Huang, X.Zhi, S.T.Fu: Med. Plants. Res Vol. 4(2010), p. 1743

[2] S.C. Ma, P.P.H. But, V.E.C. Ooi, Y.H. He, S.H.S. Lee, S.F. Lee, R.C. Lin: Biol. Pharm. Bull Vol. 24(2001), p. 311

[3] R.C. Lin, J. Peyroux, E. Seguin, M. Koch: Phytotherapy Res Vol. 5(1991), p. 188

[4] R.C.Lin, A. L.Skaltsounis, E.Seguin, F.Tillequin, M.Koch: Planta Med Vol. 60(1994), p.168

[5] C.M. Sun, W.J. Syu,Y.T. Huang, C.C. Chen, J.C. Ou: J. Nat. Prod Vol. 60(1997), p.382

[6] J.J. Chen, C.Y. Duh, J.F. Chen: Planta Med Vol. 71(2005), p.659

[7] Y.P. Lu, Y.G. Chen, J. A. Wen: Acta Botanica Yunnanica Vol. 26( 2004) , p.226

[8] Y. Cao,N.H. Tan, J.J. Chen, G.Z. Zeng, Y.B. Ma, Y.P. Wu, H. Yan, J. Yang, L.F. Lu, Q. Wang: FITOTERAPIA Vol. 81(2010), p. 253

[9] Y. Jing, A.Z. Tang, J. Liu , M. Kang, P. Deng: J Chin med mater Vol.32(2009) , p. 1864

[10] N.Y. Lee, H.Y. Min, J. Lee, J.W. Nam., Y.J. Lee, A.R. Han, A. Wiryawan, W. Suprapto, S.K. Lee, E.K. Seo: Chem Pharm Bull Vol. 56 (2008) , p. 1360

[11] K.Y. Pan, J.L. Lin, J.S. Chen: Clin Toxicol Vol.39(2001) , p. 637

[12] Y.X. Sui, S.G. Li, P.Y. Shi, Y.J. Wu, Y.X. Li, W.Y. Chen, L.Y. Huang, H. Yao, X.H. Lin: J Ethnopharmacol Vol.190(2016), p. 261

[13] Z.X. Zou, P.S. Xu, G.G. Zhang, F. Cheng, K. Chen, J. Li, W.X. Zhu, D.S. Cao, K.P. Xu, G.S. Tan: Phytochemistry Vol.134(2017), p. 114 